\title{
Three Summation Criteria for Fermat's Last Theorem
}

\section{By H. Schwindt}

Abstract. This paper extends the search for solutions of the congruences

$$
\sum_{1}^{[p / 6]} \frac{1}{i} \equiv 0, \quad \sum_{1}^{[p / 6]} \frac{1}{i^{2}} \equiv 0 \text { and } \quad \sum_{[p / 6]+1}^{[p / 5]} \frac{1}{i} \equiv 0(\bmod p)
$$

to the limit $p<600000$. The only solutions found were $p=61$ in the first case, in the second $p=205129$, and in the third case $p=109$ and $p=491$.

If the first case of Fermat's Last Theorem fails for the exponent $p$, then

$$
\sum_{1}^{[p / 6]} \frac{1}{i} \equiv 0, \quad \sum_{1}^{[p / 6]} \frac{1}{i^{2}} \equiv 0 \text { and } \sum_{[p / 6]+1}^{[p / 5]} \frac{1}{i} \equiv 0(\bmod p) .
$$

The first criterion results from theorems of Wieferich and Mirimanoff and the congruences of Lerch [1].

The second criterion results from a theorem of Vandiver and the lemma of Schwindt [2].

H. S. Vandiver and Emma Lehmer proved the third criterion [3].

For all primes $<600000$ only $p=61$ satisfies the first congruence, $p=205129$ the second, and $p=109$ and $p=491$ the third congruence. The equation

$$
i \cdot x+p \cdot y=1
$$

is equivalent to the congruence

$$
1 / i \equiv x(\bmod p)
$$

Euclid's algorithm can calculate $x$ and $y$, such that

$$
i \cdot x+p \cdot y=\operatorname{gcd}(i, p)
$$

at the same time the greatest common divisor $\operatorname{gcd}(i, p)=1$ is being calculated; see Knuth [4].

The order of this calculation is $p \cdot \log p$. The calculation-time for $p=205129$ and the first and second criterion is 29 seconds on the computer "DEC 10". For $p=600011$ it is 88 seconds.

Wilfrid Keller* has written the program for Euclid's algorithm, and he calculated for the criterion of Emma Lehmer the following data. $D$ is the average number of steps for $x$ and $t$ is the total time in seconds of the calculation $\sum 1 / i \equiv \sum x(\bmod p)$ on the computer "TR 440".

Received April 14, 1982; revised June 21, 1982.

1980 Mathematics Subject Classification. Primary 10-04.

* Rechenzentrum der Universität Hamburg. 


$\begin{array}{ccccc}p & D & D / \log p & t & t \cdot 10^{5} /(p \cdot \log p) \\ 10007 & 6.734 & 0.731 & 0.34 & 0.369 \\ 20011 & 7.324 & 0.739 & 0.64 & 0.323 \\ 30011 & 7.668 & 0.744 & 0.95 & 0.307 \\ 40009 & 7.894 & 0.745 & 1.39 & 0.328 \\ 50021 & 8.065 & 0.745 & 1.67 & 0.309 \\ 100003 & 8.695 & 0.755 & 3.48 & 0.302 \\ 500009 & 10.035 & 0.765 & 19.63 & 0.299 \\ 1000003 & 10.645 & 0.771 & 40.66 & 0.294 \\ 5000011 & 11.988 & 0.772 & 232.01 & 0.301\end{array}$

The time for the first and second criterion is five times as much. The program was run at night over half a year.

For a more cost effective criterion see Lehmer [5]. Since Lehmer's criterion [5] goes to $6 \cdot 10^{9}$, while here we go only to $6 \cdot 10^{5}$, it is clear that the foregoing gives no new information about Fermat's Last Theorem. But since the three criteria here are independent, and of interest, it seems desirable to record some data concerning them.

Rechenzentrum der Fachhochschule Hamburg

Hamburg, West Germany

1. P. Ribenboim, 13 Lectures on Fermat's Last Theorem, Springer-Verlag, Berlin and New York, 1979, pp. 155-164.

2. H. S. VANDIVER "A new type of criteria for the first case of Fermat's Last Theorem," Ann. of Math., v. 26,1925 , pp. $88-94$.

H. SCHWINDT, “Eine Bemerkung zu einem Kriterium von H. S. Vandiver," Jahresbericht d. Deutschen Math. Verein, v. 43, 1933-34, pp. 229-232.

3. EMma Lehmer, "On congruences involving Bernoulli numbers and the quotients of Fermat and Wilson,” Ann. of Math., v. 39, 1938, pp. 350-359.

4. D. E. KNuth, The Art of Computer Programming, Vol. 2, Seminumerical Algorithms, Addison-Wesley, Reading, Mass., 1973, p. 325.

5. D. H. Lehmer, “On Fermat's quotient, base two,” Math. Comp., v. 36, 1981, pp. 289-290. 